Draft VERSion OCtOBER 2, 2020

Typeset using LATEX RNAAS style in AASTeX62

\title{
Two Nested SHells AROUnd The BLue supergiant ALS 19653
}

\author{
J.M. Drudis ${ }^{1}$ and V.V. Gvaramadze ${ }^{2,3}$ \\ ${ }^{1}$ Curiosity2 Observatory, New Mexico Skies, Mayhill, NM 88339, USA \\ ${ }^{2}$ Sternberg Astronomical Institute, Lomonosov Moscow State University, Universitetsky pr., 13, Moscow 119234, Russia \\ ${ }^{3}$ Space Research Institute, Russian Academy of Sciences, Profsoyuznaya 84/32, 117997 Moscow, Russia
}

Keywords: circumstellar matter — stars: individual (ALS 19653) — stars: massive — supergiants

\begin{abstract}
We present the results of deep narrowband imaging of two nested shells around the blue supergiant ALS 19653, which confirm that the outer shell is physically associated with the star.

\section{INTRODUCTION}

Massive stars experience episodes of intense mass loss during their lifetime, leading to the formation of parsec-scale circumstellar nebulae of various forms. Searching for such nebulae serves as an effective way to detect rare types of massive stars, such as the luminous blue variables, blue supergiants and Wolf-Rayet stars (e.g. Gvaramadze et al. 2010, 2012). One of the massive stars revealed in this way is the central star, ALS 19653, of a dumbbell-like infrared nebula discovered (Gvaramadze et al. 2019, hereafter Paper I) in the archival data of the Wide-field Infrared Survey Explorer (WISE; Wright et al. 2010). Follow-up inspection of $\mathrm{H} \alpha+[\mathrm{N}$ II] images from the Isaac Newton Telescope (INT) Photometric H $\alpha$ Survey of the Northern Galactic Plane (IPHAS; Drew et al. 2005) lead to the detection of an almost circular shell (of angular radius of $\approx 0.5$ arcmin or $\approx 0.2 \mathrm{pc}$ ) surrounding the infrared nebula and a more extended and faint shell-like structure (of angular radius of $\approx 2.7$ arcmin or $\approx 1.2 \mathrm{pc}$ ) also centered on ALS 19653 (Paper I). Subsequent optical spectroscopic observations showed that ALS 19653 is a B0.5 supergiant, and allowed to derive the expansion velocities of the inner and outer shells of $\approx 20-30$ and $100 \mathrm{~km} \mathrm{~s}^{-1}$, respectively (Paper I). It was also found that the spectrum of the inner shell shows only emission lines of $\mathrm{H} \alpha$ and $[\mathrm{N} \mathrm{II}] \lambda \lambda 6548,6584$, which is consistent with the low expansion velocity of this shell. On the other hand, the higher expansion velocity derived for the outer shell implies that this shell could be a source of [O III] emission. The main purpose of the study presented in this note is the deep narrowband imaging of the environs of ALS 9653, aimed to get a better idea of the morphology of the outer shell and to confirm its connection to the star.
\end{abstract}

\section{NARROWBAND IMAGING}

To fulfill our purpose, a deep color image was planned to be taken with the $61 \mathrm{~cm}$ telescope (f/6.5; camera FLI PL16803 16MPx, 9 $\mu$ ) at the Curiosity2 Observatory using narrowband (FWHM $15 \AA) \mathrm{H} \alpha$, [N II] and [O III] filters centered, respectively, at 6563,6584 and $5007 \AA$. The exposure times for individual images taken with these filters were set at $40 \mathrm{~min}$ each. After taking $37 \mathrm{H} \alpha$ and 26 [O III] images (total exposure times of $\approx 25$ and 17 hours, respectively), it became clear that, on one side, our $\mathrm{H} \alpha$ image (Figure 1(b)) did not lack any detail that was already present in the IPHAS image. This was a strong indication that there was no specific [N II] emission in this nebula, and we then dropped the capture of any frames with the $[\mathrm{N} \mathrm{II}]$ filter. The second conclusion was that the [O III] images did not show any traces of neither the inner nebula (which is consistent with the lack of the [O III] $\lambda 5007$ line in its spectrum; see Paper I) nor the outer shell. Correspondingly, for the final color image (Figure 1(a)) we used only the $\mathrm{H} \alpha$ (red) image and a set of short exposure frames through the broadband $R, G$ and $B$ filters in order to depict the stars in the image with their true natural colors. The processing of this image was very simple in order to preserve the original structures. The only treatment was a dual deconvolution applied on different parts of the image. A maximum entropy

Corresponding author: J.M. Drudis

astrodrudis2016@gmail.com 
deconvolution was applied to the outer shell, in order to enhance its presence, and a positive constraint deconvolution was applied to the central nebula in order to obtain greater detail of its structure. Figure 1(b) shows the outer shell without applying the maximum entropy deconvolution, in order to show its real brightness, compared to the central nebula.

Figure 1(a) shows that the outer shell represents an almost circular nebula whose emission fills nearly the entire space between its outer edge and the central nebula. One can also see that the maximum brightness of the shell falls on its north-east edge and that ALS 19653 is somewhat offset towards this edge. This could be caused either by a north-east motion of the star relative to the ambient medium or by a density gradient in the same direction.

\section{SPACE VELOCITY OF ALS 19653}

To check whether the space motion of ALS 19653 is the cause of the increased brightness of the north-east edge of the outer shell, we calculated the peculiar transverse velocity, $v_{\mathrm{tr}}$, of this star using its Gaia DR2 proper motion $\left(\mu_{\alpha} \cos \delta=-0.188 \pm 0.113\right.$ mas yr $^{-1}, \mu_{\delta}=-2.203 \pm 0.112$ mas yr $\left.^{-1}\right)$ and parallax $(0.639 \pm 0.063$ mas $)$ measurements (Gaia Collaboration et al. 2018). To do this, we adopted the solar Galactocentric distance of $R_{0}=8.0 \mathrm{kpc}$ and the circular Galactic rotation velocity of $\Theta_{0}=240 \mathrm{~km} \mathrm{~s}^{-1}$ (Reid et al. 2009), and the solar peculiar motion of $\left(U_{\odot}, V_{\odot}, W_{\odot}\right)=(11.1,12.2,7.3) \mathrm{kms}^{-1}$ (Schönrich et al. 2010). We derived the peculiar velocity components along the Galactic longitude and latitude of $v_{1}=8.5 \pm 0.8 \mathrm{~km} \mathrm{~s}^{-1}$ and $v_{\mathrm{b}}=1.4 \pm 0.8 \mathrm{~km} \mathrm{~s}^{-1}$, respectively, which correspond to $v_{\mathrm{tr}}=8.6 \pm 0.8 \mathrm{~km} \mathrm{~s}^{-1}$. Using the systemic radial velocity of ALS 19653 of $-9.0 \pm 0.1 \mathrm{~km} \mathrm{~s}^{-1}$ (Paper I), we calculated also the peculiar radial velocity $v_{r}=-17.6 \pm 0.1 \mathrm{~km} \mathrm{~s}^{-1}$ and the total space velocity of this star of $v_{\text {tot }}=19.5 \pm 0.36 \mathrm{~km} \mathrm{~s}^{-1}$. The quoted uncertainties in the velocity estimates take into account only the uncertainties in the proper motion and systemic velocity measurements. Although the derived value of the space velocity did not allow us to treat ALS 19653 as a classical runaway star, its orientation in the sky shows that this star is moving in the "correct" (north-east) direction, meaning that the enhanced brightness of the north-east edge of the outer shell could indeed be caused by this motion.

\section{DISCUSSION}

Detection of two nested shells around ALS 19653 indicates that this star at least twice went through episodes of enhanced mass lose in the recent $\left(\sim 10^{4} \mathrm{yr}\right)$ past. Similar two-component nebulae were also found around both massive and low-mass stars, e.g., the O6f?p star HD 148937 (Leitherer \& Chavarria-K 1987) and the M1 II star HD 65750 (Drudis 2018). The common characteristic of these objects is that their inner nebulae possess axial symmetry. Although the mechanisms that lead to the formation of such nebulae are not fully understood, it is believed that they are somehow related to the present or past binarity of their central stars. In particular, the fast rotation and strong magnetic field of HD 148937 and the nitrogen-rich composition of its inner nebula make this star a strong merger candidate (Langer 2012). Similarly, it was suggested that ALS 19653 is a merger product as well (Paper I). The possible binarity of these two stars (Wade et al. 2019; Gvaramadze et al. 2019) does not contradict these suggestions because both stars could originally be members of triple or higher multiple systems (cf. Langer 2012).

Finally, we note that a careful inspection of the WISE $22 \mu \mathrm{m}$ image revealed that the central nebula is surrounded by a diffuse halo of the same angular extent as the outer optical shell (Figures 1(c) and 1(d)), which provides further evidence of the connection of this shell with ALS 19653.

V.V.G. acknowledges support from the Russian Foundation for Basic Research grant 19-02-00779. 


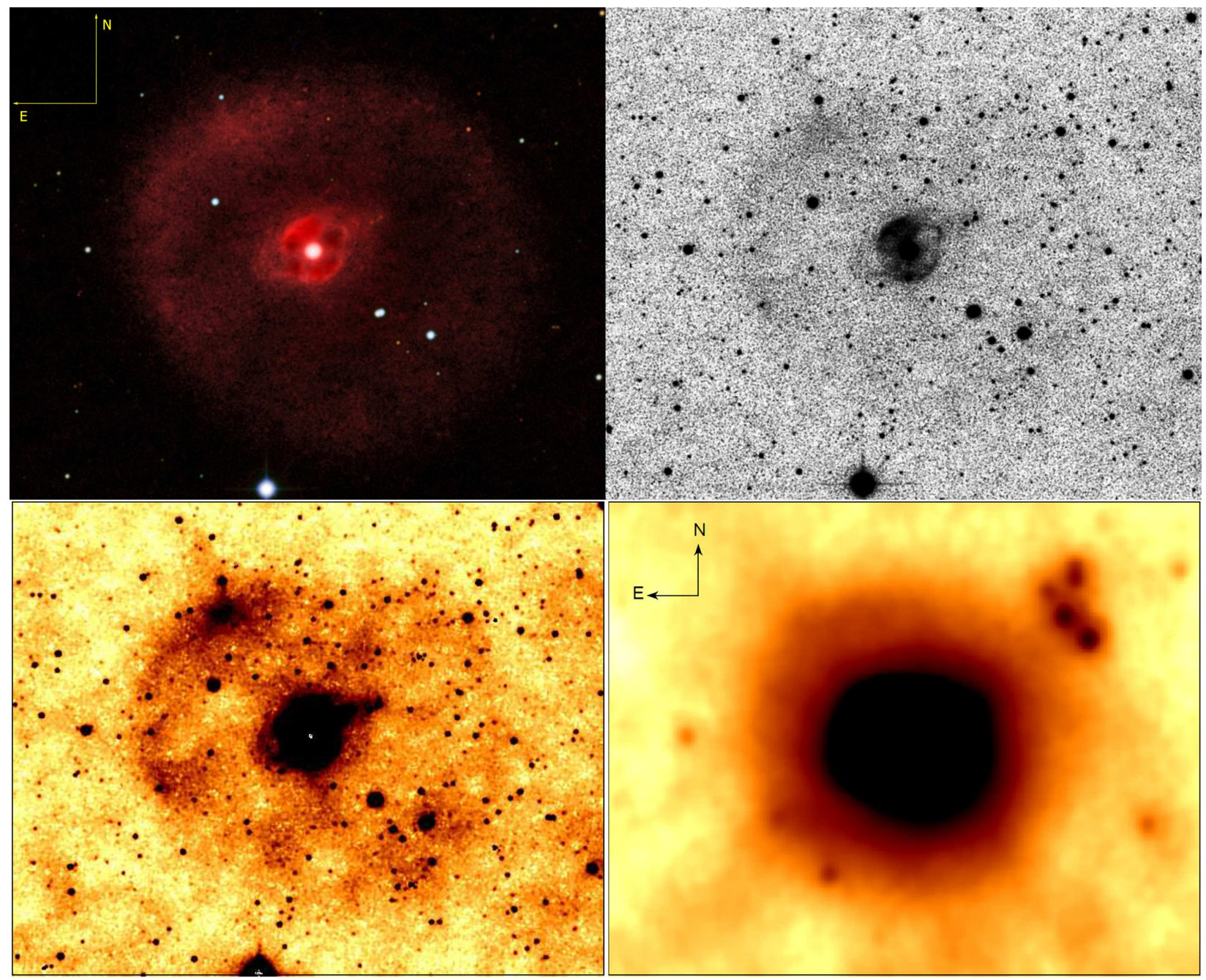

Figure 1. Figure 1(a) (upper left): final color image. Figure 1(b) (upper right): inverted H $\alpha$ image. Figures 1(c) and 1(d) (bottom panels): $\mathrm{H} \alpha$ (left) and WISE $22 \mu \mathrm{m}$ (right) images. The orientation and the scale of all images are the same.

\section{REFERENCES}

Drew J., Greimel R., Irwin M. J. et al 2005 MNRAS 362 753

Drudis J. 2018 RNAAS 241

Gaia Collaboration, Brown A. G. A., Vallenari A. et al 2018 A\&SA 616 A1

Gvaramadze V. V., Kniazev A. Y. and Fabrika S. 2010 MNRAS 4051047

Gvaramadze V. V., Kniazev A. Y., Miroshnichenko A. S. et al 2012 MNRAS 4213325

Gvaramadze V. V., Kniazev A. Y., Castro N. and Grebel E. K. 2019 AJ 15753
Langer N. $2012 A R A \& A 50107$

Leitherer C. and Chavarria-K C. 1987 A\&A A 175208

Reid M. J., Menten K. M., Zheng X. W., Brunthaler A. and Xu Y. 2009 ApJ 7051548

Schönrich R., Binney J. and Dehnen W. 2010 MNRAS 403 1829

Wade G. A., Smoker J. V., Evans C. J. et al 2019 MNRAS 4832581

Wright E. L., Eisenhardt P. R. M., Mainzer A. K. et al 2010 AJ 1401868 